\title{
Caracterización mecánica de un acero ferrítico/martensítico de activación reducida de producción española ${ }^{(\cdot)}$
}

\author{
D. Rodríguez* y M. Serrano**
}

\begin{abstract}
Resumen
En este documento se reflejan los primeros resultados referentes a la caracterización de dos coladas de un acero 9 Cr ferrítico/martensítico de activación reducida (RAFM) producido en España, denominadas AF1B y AF2A. Los resultados obtenidos en dicha caracterización se compararán con su homólogo europeo, el EUROFER97-2, el cual se eligió como material de referencia. Todas las actividades detalladas se realizaron en la Unidad de Materiales Estructurales del CIEMAT, dentro del proyecto TECNO-FUS CONSOLIDER INGENIO. Ambas coladas, producidas en una planta piloto de la Fundación ITMA, presentan el mismo proceso de producción y tratamiento térmico final. Asimismo presentan un comportamiento a tracción similar al del EUROFER97-2. Por el contrario, las propiedades a impacto son menores a las del homólogo europeo. En la microestructura de la colada AF1B se han detectado inclusiones bifásicas de gran tamaño que afectan a sus propiedades mecánicas, sobre todo a las de impacto. Estas inclusiones no se detectaron en la AF2A.
\end{abstract}

\section{Mechanical characterization of a reduced activation $9 \mathrm{Cr}$ ferritic/martensitic steel of spanish production}

\begin{abstract}
This paper shows the first results concerning the characterization of two heats of a reduced activation $9 \mathrm{Cr}$ ferritic/martensitic steel (RAFM) made in Spain, called AF1B and AF2A. The results of this characterization are compared with their European counterparts, EUROFER97-2, which was chosen as reference material. All activities described were performed in the Structural Materials Unit of CIEMAT, within the national project TECNO-FUS CONSOLIDER INGENIO.The two Spanish heats have the same production process and heat treatment. Both heats have a similar tensile behaviour similar to EUROFER97-2, but on the other hand impact properties are lower. The microstructure of AF1B reveals large biphasic inclusions that affecting its mechanical properties, especially the impact properties. AF2A casting was free of these inclusions.
\end{abstract}

Keywords

Reduced activation ferritic/martensitic steel; Biphasic inclusions; Mechanical properties; Microstructure.

\section{INTRODUCCIÓN}

En las últimas décadas el incremento exponencial del consumo energético, junto con la búsqueda de nuevas plantas de generación de energía cada vez más seguras y longevas, ha propiciado el desarrollo de las nuevas generaciones de reactores de fisión (GEN III y GEN IV), la construcción del primer reactor experimental de fusión termonuclear, denominado ITER (International Thermonuclear Experimental Reactor), junto con el primer prototipo de sistema de transmutación de residuos radioactivos asistido por acelerador, denominado MYRRHA. Pero para que esta nueva era energética vea la luz, es necesario el desarrollo de una nueva generación de materiales tanto estructurales como funcionales que sean capaces de operar de forma efectiva y viable en las nuevas condiciones de servicio que se plantean, debido a que en la mayoría de los diseños de estos nuevos reactores, combinan altas dosis de radiación neutrónica con elevadas temperaturas y presencia de metales líquidos ${ }^{[1]}$.

En el caso de los materiales estructurales para fusión, se maneja el concepto de materiales de activación reducida (reduced-activation materials, RAFM), capaces de obtener niveles aceptables de actividad

(•) Trabajo recibido el día 6 de diciembre de 2011 y aceptado en su forma final el día 14 de marzo de 2012 .

* Laboratorio Nacional de Fusión. CIEMAT, Avda de la Complutense 40, 28040 Madrid.

** División de materiales estructurales CIEMAT, Avd.a de la Complutense 40, 28040 Madrid. 
en pocos cientos de años ${ }^{[2]}$. La base de este tipo de aceros se centra en la naturaleza de sus aleantes, ya que pocos elementos pueden cumplir el requisito de actividad reducida, entre los más importantes se pueden encontrar el cromo, vanadio, titanio, wolframio y el tántalo, dichos elementos poseen tiempos de activación radiológica relativamente $\operatorname{cortos}^{[3]}$.

Los aceros RAFM se desarrollaron a partir de los aceros ferríticos/martensíticos (F/M) convencionales, procedentes tanto de la industria térmica como de los programas de reactores rápidos (T91, JMFS...), debido, no solo a sus buenas propiedades como materiales estructurales, sino también por su mayor resistencia al hinchamiento por irradiación, denominado en inglés "Swelling"[4-6]. Los principales aceros RAFM, existentes en la actualidad son: el denominado F82HMod, producido por la NKK Corporation ${ }^{[7]}$ y el acero EUROFER97 producido en el marco de la European Fusion Development Agreement (EFDA) ${ }^{[8]}$. En la actualidad, se sigue trabajando en la optimización de estos materiales (F82H-BA07, F82 Hmod3, EUROFER $97-2$ y -3$)^{[9}$ y 10], y además existen nuevas aleaciones RAFM procedentes de China e India ${ }^{[11}$ y 12$]$.

Esta es una de las razones por las cuales se desarrolla en el marco del proyecto español TECNO-FUS CONSOLIDER INGENIO, y más concretamente en las tareas 4.1 y 4.4 , un acero RAFM a escala semi-industrial $100 \%$ español. En la actualidad ya existen varias coladas experimentales de este RAFM español bajo estudio. En este documento se muestran los primeros resultados tanto microestructurales como mecánicos.

\section{MATERIALES Y PROBETAS}

Las coladas en estudio han sido fabricadas por la Fundación ITMA en el marco del proyecto TECNO-FUS [13]. Dichas coladas se obtuvieron por medio de un horno de alto vacío, en inglés Vacuum
Induction Meeting (VIM), con capacidad para unos $50 \mathrm{~kg}$ aproximadamente. Tras el proceso de fundición cada colada fue desbastada y dividida en dos piezas rectangulares denominadas "slabs" de 250x125x100 mm, las cuales se sometieron a un proceso de laminado en caliente hasta obtener placas de $14 \mathrm{~mm}$ de espesor. Tras el proceso de laminado fueron sometidas al mismo tratamiento térmico (TT). La única diferencia importante estriba en el \% en peso de wolframio, en base a lo cual se denominó AF1B a la colada con $1,4 \%$ y AF2A a la de $1,08 \%$.

Además de estas coladas experimentales también se analizaron las propiedades mecánicas de una placa de $25 \mathrm{~mm}$ de espesor de EUROFER97-2, existente en el CIEMAT y fabricado en el marco de EFDA, ya que es el material de referencia elegido, por las razones anteriormente detalladas. Dicho RAFM presenta un tratamiento térmico diferente al de las coladas AF2A y AF1B. Tanto los TT como las composiciones químicas de cada RAFM pueden verse en las tablas I y II.

Previa a la caracterización mecánica, se realizó un examen microestructural preliminar, el cual se centró en las técnicas de microscopía óptica y microscopía electrónica de barrido (SEM). En cuanto a la caracterización mecánica, incluye ensayos de tracción en las orientaciones longitudinal (L) y transversal (T), es decir, en la dirección principal de laminación $\mathrm{L}$, y en la dirección perpendicular a la de laminación T (Fig. 1).

También se realizaron ensayos de impacto Charpy con probetas estándar CVN en orientación TL, y probetas miniatura del tipo KLST en orientaciones LT y $\mathrm{TL}$, ver figuras 2 y 3.

El modo de nombrar a las probetas de impacto corresponde a la norma ASTM $399^{[14]}$, en la cual la primera letra define la dirección principal de mecanizado y la segunda la dirección de propagación de la grieta.

Los ensayos de tracción se han realizado en una máquina universal de ensayos MTS de $100 \mathrm{kN}$ de capacidad, siguiendo la norma ASTM E8 ${ }^{[15]}$. Los

Tabla I. Tratamiento térmico de cada RAFM

Table I. Heat treatment of each RAFM

\begin{tabular}{lcc}
\hline & RAFM ESPAÑOL & EUROFER 97-2 \\
\hline Normalizado & $\begin{array}{c}960^{\circ} \mathrm{C} \\
30 \mathrm{~min} / \text { aire }\end{array}$ & $\begin{array}{c}960^{\circ} \mathrm{C} \\
1,5 \mathrm{~h} / \text { aceite }\end{array}$ \\
\hline Revenido & $750^{\circ} \mathrm{C}$ & $750^{\circ} \mathrm{C}$ \\
& $2 \mathrm{~h} /$ aire & 4 h/aire \\
\hline
\end{tabular}


Tabla II. Composición química de las coladas experimentales de RAFM de producción nacional

Table II. Chemical composition of experimental casting RAFM of national production

\begin{tabular}{|c|c|c|c|c|}
\hline & & \multicolumn{2}{|c|}{ RAFM español } & \multirow{2}{*}{$\begin{array}{c}\text { EUROFER97-2 } \\
\text { (\% Peso) }\end{array}$} \\
\hline & & $\begin{array}{c}\text { AF1B } \\
\text { (\% Peso) }\end{array}$ & $\begin{array}{c}\text { AF2A } \\
\text { (\% Peso) }\end{array}$ & \\
\hline \multirow[t]{9}{*}{ A } & $\mathrm{Cr}$ & 8,9 & 8,8 & 8,85 \\
\hline & C & 0,12 & 0,12 & 0,092 \\
\hline & $\mathrm{Mn}$ & 0,39 & 0,44 & 0,44 \\
\hline & $\mathrm{P}$ & 0,004 & 0,003 & 0,013 \\
\hline & S & 0,007 & 0,005 & $<0,003$ \\
\hline & V & 0,19 & 0,19 & 0,18 \\
\hline & $\mathrm{B}$ & 0,001 & $<0,002$ & $<0,001$ \\
\hline & $\mathrm{N}$ & 0,015 & 0,007 & 0,018 \\
\hline & 0 & $<0,01$ & 0,02 & 0,0008 \\
\hline \multirow[t]{3}{*}{ B } & W & 1,43 & 1,08 & 1,08 \\
\hline & $\mathrm{Ta}$ & 0,1 & 0,12 & 0,15 \\
\hline & $\mathrm{Ti}$ & 0,004 & 0,004 & 0,001 \\
\hline \multirow[t]{11}{*}{ C } & $\mathrm{Nb}$ & 0,004 & $<0,005$ & $<0,002$ \\
\hline & Mo & 0,005 & 0,005 & $<0,01$ \\
\hline & $\mathrm{Ni}$ & 0,01 & 0,01 & 0,07 \\
\hline & $\mathrm{Cu}$ & 0,015 & 0,01 & 0,04 \\
\hline & $\mathrm{Al}$ & 0,01 & 0,05 & 0,002 \\
\hline & $\mathrm{Si}$ & 0,05 & 0,04 & 0,04 \\
\hline & Co & 0,008 & 0,006 & 0,02 \\
\hline & $\mathrm{Sn}$ & 0,03 & 0,006 & $<0,002$ \\
\hline & As & & 0,01 & $<0,002$ \\
\hline & $\mathrm{Sb}$ & & 0,01 & 0,003 \\
\hline & $\mathrm{Zr}$ & & 0,002 & $<0,0005$ \\
\hline
\end{tabular}

A: Elementos aleantes.

B: Elementos de sustitución.

C: Elementos radiológicamente indeseables.

ensayos se han realizado entre temperatura ambiente y $700{ }^{\circ} \mathrm{C}$ a una velocidad de $0,001 \mathrm{~mm} / \mathrm{mm} \cdot \mathrm{min}^{-1}$. En la realización de los ensayos de impacto con probetas estandarizadas se ha utilizado un péndulo WOLPERT de $300 \mathrm{~J}$ de capacidad, bajo la norma ASTM 23 [16]. En el caso de los las probetas subdimensionadas se usó un péndulo también WOLPERT de $50 \mathrm{~J}$ de capacidad. El intervalo de temperaturas usado en el caso de las probetas estándar estuvo entre los $-115^{\circ} \mathrm{C}$ y los $100^{\circ} \mathrm{C}$, en el caso de las probetas miniatura KLST estuvo entre los $-150^{\circ} \mathrm{C}$ y ambiente.

La dureza se ha medido con un durómetro de la marca AKASHI-II, dotado con una punta de dia- mante del tipo Vickers. La carga aplicada para cada una de las penetraciones fue de $30 \mathrm{~kg}$.

\section{RESULTADOS}

La caracterización microestructural se ha realizado en los tres planos principales de la placa (LT, LS y TS) ${ }^{[14]}$, para analizar la posible anisotropía del material. Las micrografías se pueden observar en la figura 4.

Como podemos ver en el esquema tridimensional de cada material, todos muestran la estructura típica de un acero ferrítico/martensítico, o lo que 


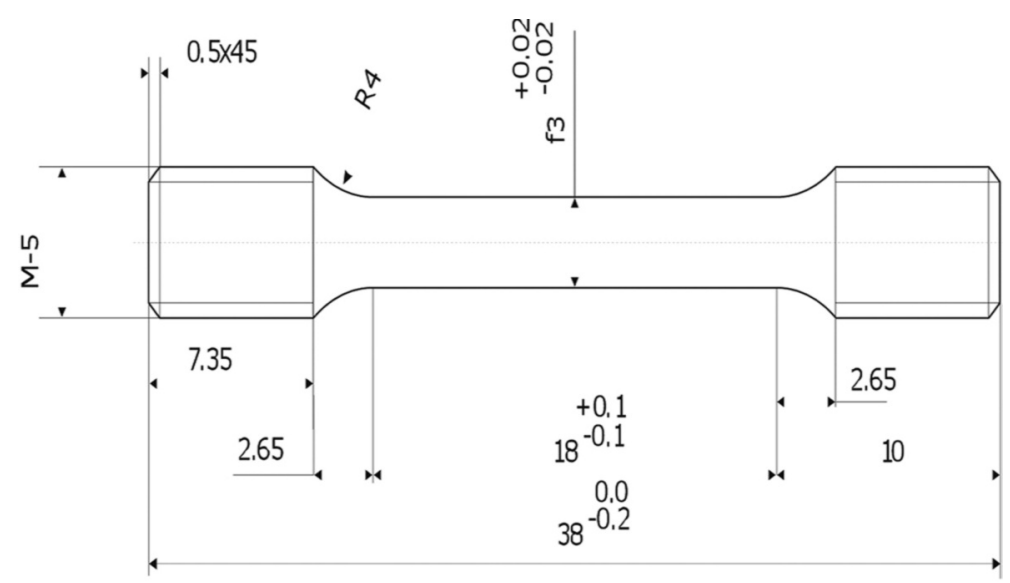

Figura 1. Plano de una probeta de tracción de $\mathrm{M} 5(\mathrm{~mm})$.

Figure 1. Drawing of a M5 tensile specimen $(\mathrm{mm})$.
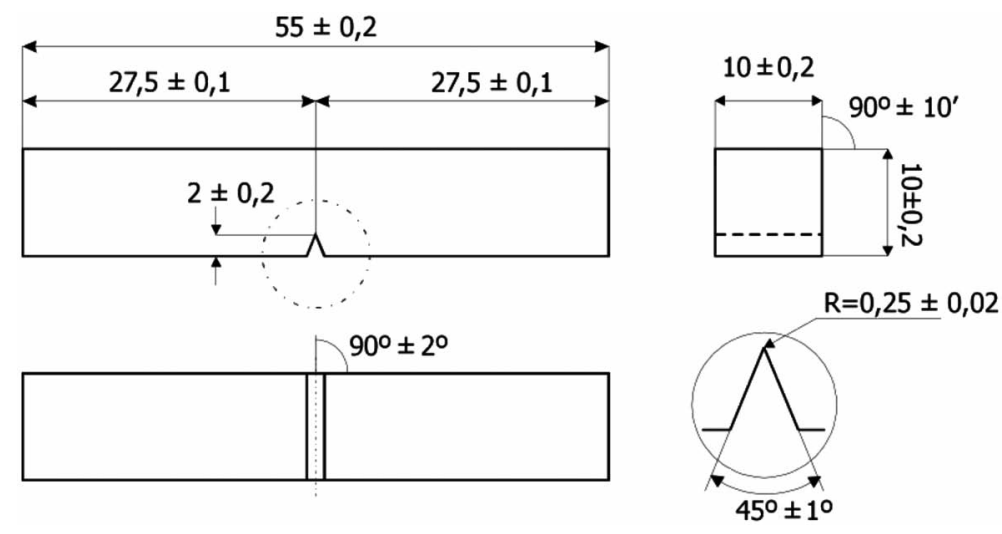

Figura 2. Plano de una probeta estandarizada CVN para ensayos de impacto $(\mathrm{mm})$.

Figure 2. Plan of a CVN standard specimen for testing impact ( $\mathrm{mm})$.
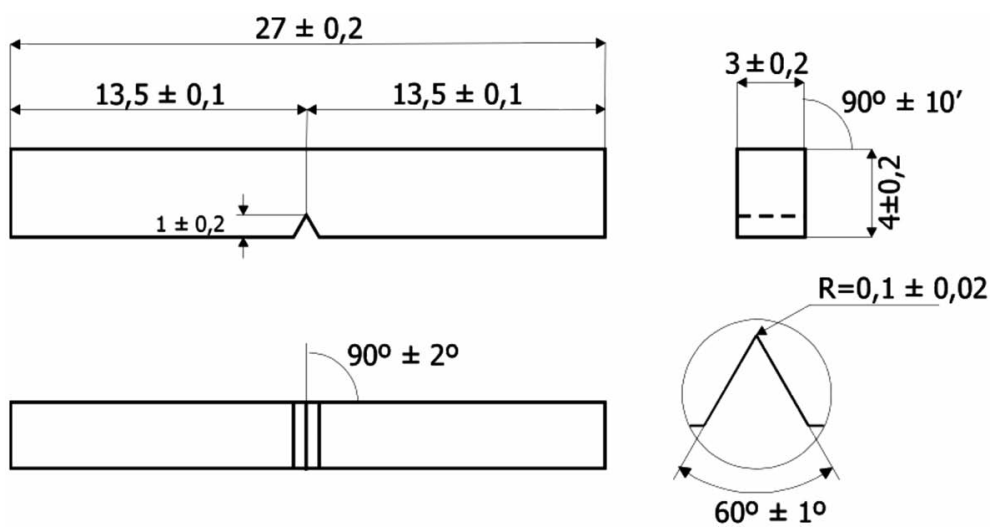

Figura 3. Plano de una probeta subdimensionada KLST para ensayos de impacto $(\mathrm{mm})$.

Figure 3. Plan of a KLST non-standard specimen for testing impact $(\mathrm{mm})$. 


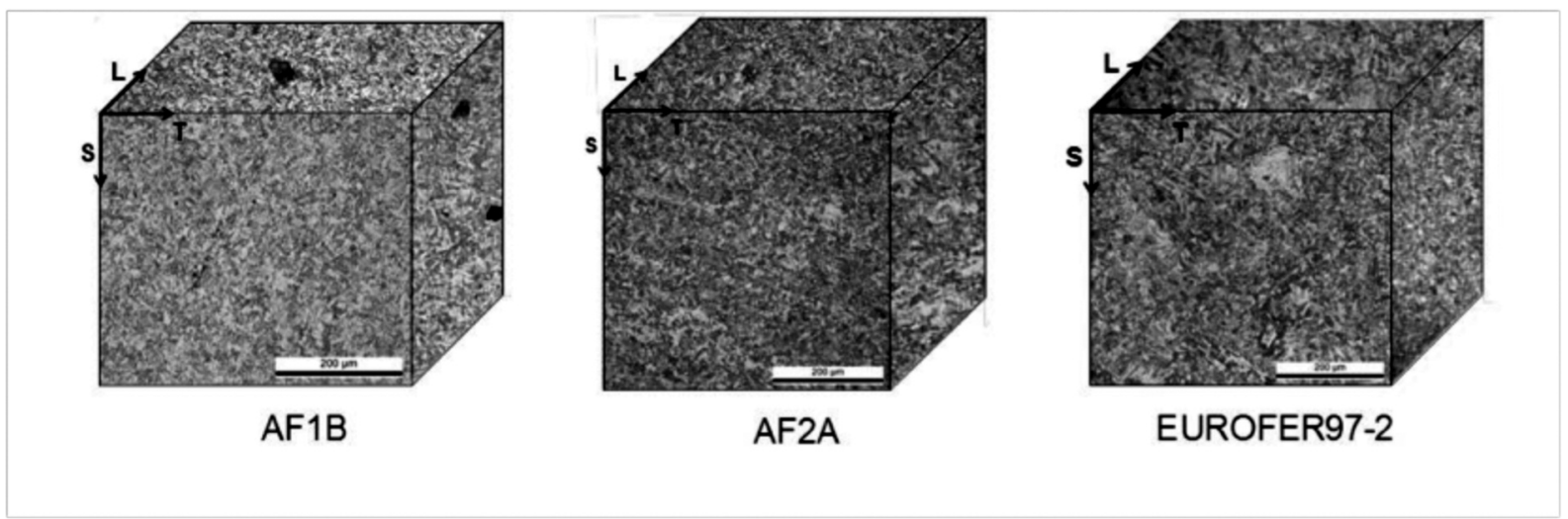

Figura 4. Imagen por microscopia óptica de los diferentes RAFM bajo estudio (200 $\mu \mathrm{m})$.

Figure 4. Optical microscopy image of different RAFM under study (200 $\mu \mathrm{m})$.

es lo mismo, la fase martensita provoca que la anisotropía generada durante el proceso de laminación sea, a nivel microestructural, muy leve.

Por otro lado al ir a más aumentos (Fig. 5), podemos ver como el EUROFER97-2 posee unas placas de martensita algo mayores que las observadas en las coladas AF1B y AF2A.

Esta primera impresión se confirma a la hora de medir el tamaño de grano austenítico primario de cada material según la norma ASTM E112-96 ${ }^{\mathrm{E} 3}$, ya que el EUROFER97-2 posee un tamaño de grano en torno a los 7,9 y $11 \mu \mathrm{m}$ (según norma 10-11ASTM) mientras que para las coladas AF1B y AF2A se encuentra entre las 5,6 y 7,9 $\mu \mathrm{m}$ (según norma 11-12ASTM) ${ }^{[17]}$. Además de un tamaño de grano diferente entre el EUROFER97-2 y nuestro RAFM experimental, también hemos observado la existencia de una gran cantidad de inclusiones con diferente morfología y tamaño en la colada AF1B. Estas inclusiones no se han observado ni en la colada AF2A, ni en el EUROFER97-2, al menos no en tamaños y concentraciones similares (Figs. 4 y 5), por lo que pueden ser responsables de las diferencias en el comporta- miento mecánico entre ambas coladas, como podremos ver más adelante.

Tras este examen preliminar de la microestructura, se procedió a analizar su dureza mediante ensayos de punzonado del tipo Vickers. Las medidas obtenidas en cada material se encuentran dentro de los límites dados por EFDA para el EUROFER97-2 $(200-240 \mathrm{HV})^{[18]}$. Además no presentan discontinuidades importantes entre caras. Dichos valores se pueden ver en la tabla III

Tabla III. Resultados del ensayo de dureza HV30

Table III. Results of HV30 hardness testing

\begin{tabular}{ccc}
\hline \multicolumn{3}{c}{ HV30 } \\
\hline EUROFER97-2 & AF1B & AF2A \\
\hline 232 & 230 & 224 \\
\hline
\end{tabular}

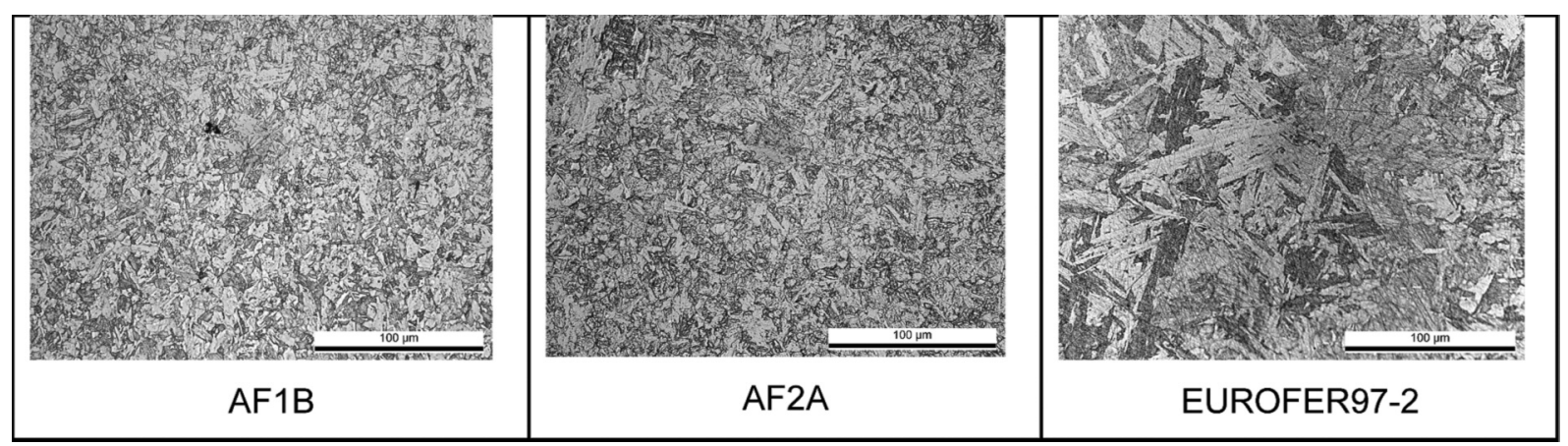

Figura 5. Detalle por microscopia óptica del plano LT de cada RAFM.

Figura 5. Optical microscopic detail at the plane LT of each RAFM. 
CARACTERIZACIÓN MECÁNICA DE UN ACERO FERRITICO/MARTENSÍTICO DE ACTIVACIÓN REDUCIDA DE PRODUCCIÓN ESPAÑOLA MECHANICAL CHARACTERIZATION OF A REDUCED ACTIVATION 9CR FERRITIC/MARTENSITIC STEEL OF SPANISH PRODUCTION

A continuación podemos ver en la tabla IV los resultados obtenidos en los ensayos de tracción para la resistencia (Ultimate Tensile Strength UTS), el límite elástico $\left(\sigma_{\mathrm{YS} 0,2 \%}\right)$, la estricción y la elongación en las temperaturas críticas según la especificaciones mecánicas del EUROFER97-2 dadas por EFDA.

En las figuras 6 y 7 , podemos ver la variación de algunos de estos valores en función de la temperatura, para los materiales bajo estudio.

Como podemos ver, las dos coladas experimentales presentan un comportamiento frente a la temperatura muy similar. Además satisfacen la especificación del EUROFER97-2 por la cual se exige que posean un $\sigma_{\text {YS } 0,2 \%}$ superior a $500 \mathrm{MPa}$ a temperatura ambiente y a $300 \mathrm{MPa}$ a $550^{\circ} \mathrm{C}$, además de una UTS a temperatura ambiente superior a los $600 \mathrm{MPa}$. Al comparar estos valores con los obtenidos para el EUROFER97-2, vemos como la colada AF2A es la que en un principio presenta unas propiedades a tracción ligeramente inferiores, mientras que la resistencia mecánica de la colada AF1B es ligeramente superior a la del EUROFER97-2. Estas diferencias desaparecen a temperaturas cercanas a las de operación, unos $500^{\circ} \mathrm{C}$.

Esta similitud en el comportamiento mecánico entre los RAFM analizados se refleja también en el porcentaje de estricción, ya que como podemos ver en la figura 7, la reducción de área obtenida para cada material es prácticamente idéntica. No obstante, en la tabla IV podemos ver cómo la colada AF1B presenta valores de fractura dúctil, reflejados en la estricción y en la elongación final, ligeramente menores que los obtenidos para la colada AF2A.

En la figura 8 podemos ver la curva de transición con probetas Charpy estándar tipo CVN para las coladas AF2A y AF1B, junto a sus respectivos ajustes (Tabla V) ${ }^{[16]}$, donde USE es el nivel de energía superior, LSE es el nivel de energía inferior, $\mathrm{T}_{\mathrm{M}}$ es la temperatura para la cual la energía absorbida es de (USE-LSE)/2, también conocida como temperatura de transición

Tabla IV. Valores críticos y de especificación del ensayo de tracción

Table 4. Critical and specification values of the tensile test

\begin{tabular}{lccccc}
\hline & UTS (MPa) & \multicolumn{2}{c}{$\sigma Y S \mathbf{( M P a )}$} & Estricción (\%) & Elongación (\%) \\
\cline { 2 - 7 } & $\mathbf{2 0}{ }^{\circ} \mathbf{C}$ & $\mathbf{2 0}{ }^{\circ} \mathbf{C}$ & $\mathbf{5 0 0}{ }^{\circ} \mathbf{C}$ & Ambiente & Ambiente \\
\hline EUROFER97-2 & 670 & 542 & 382 & 78,5 & 26,5 \\
AF1B & 675 & 531 & 387 & 76,6 & 27,0 \\
AF2A & 646 & 527 & 382 & 77,5 & 28,9 \\
Especificación & $>600$ & $>500$ & $>300$ & - & $>15 \%$ \\
\hline
\end{tabular}
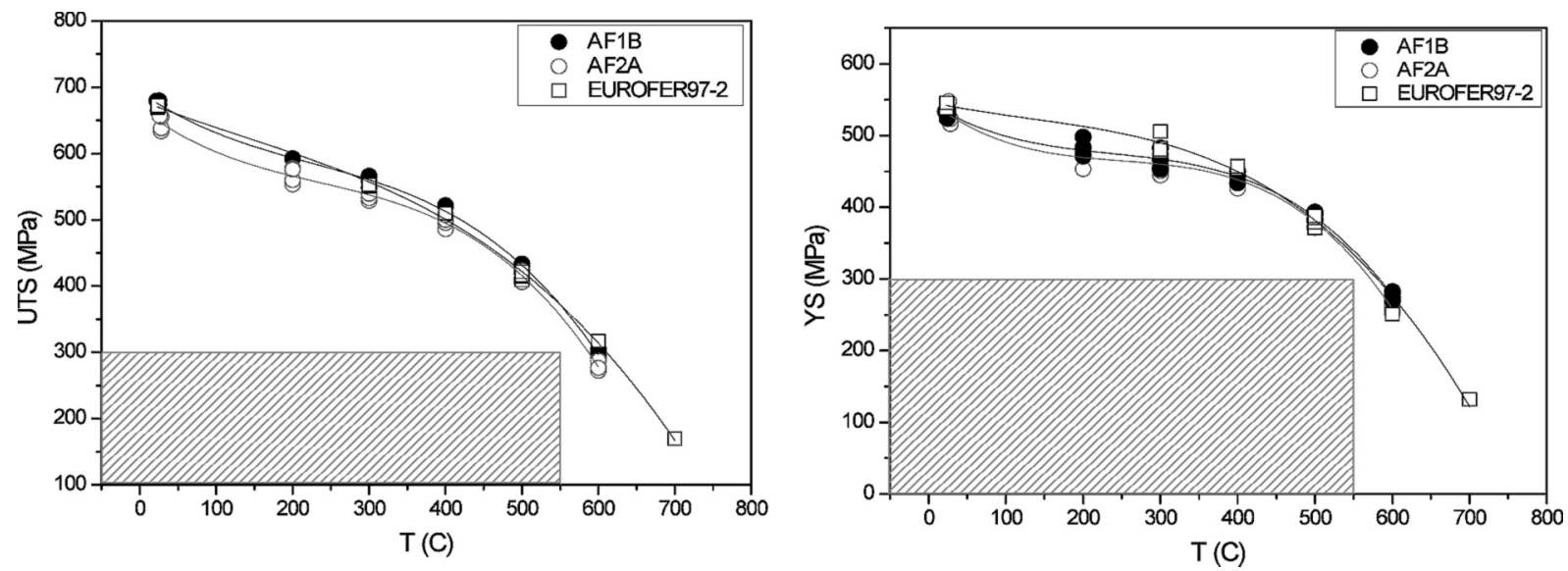

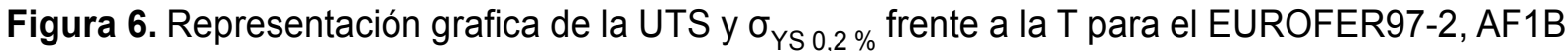
y AF2A.

Figure 6. Graphic representation of the UTS and $\sigma_{Y S 0.2 \%}$ vs T to EUROFER97-2, AF1B and AF2A. 


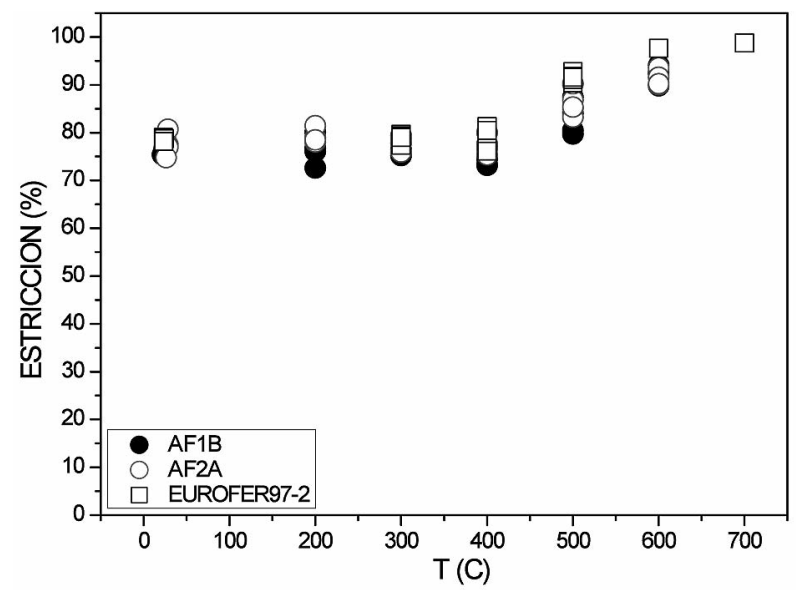

Figura 7. Representación grafica del porcentaje de estricción del EUROFER97-2, AF1B y AF2A.

Figura 7. Graphic representation of necking vs $T$ for EUROFER97-2, AF1B and AF2A.

Tabla V. Valores del ajuste de la curva E vs T

Table V. Values of fitting the curve $E$ vs $T$

\begin{tabular}{lccc}
\hline & USE (J) & LSE (J) & DBTT $\left({ }^{\circ} \mathbf{C}\right)$ \\
\hline AF1B & 164,9 & 7,8 & $-49,6$ \\
AF2A & 203,3 & 0,0 & $-67,4$ \\
\hline
\end{tabular}

dúctil-frágil (Ductile to Brittle Transition Temperatura; DBTT) y C representa el ancho de la zona de transición.

El análisis de cada curva refleja que la colada AF1B no solo tiene una USE mucho más baja que la AF2A, sino que entre ambas coladas existe una diferencia de casi $20^{\circ} \mathrm{C}$ en la DBTT. Estos valores no reflejan la similitud esperada a la hora de comparar dos aceros que han sido sometidos a procesos de laminado en caliente bajo las mismas condiciones, y que, posteriormente, han recibido el mismo tratamiento térmico. Además las diferencias existentes entre ambas coladas referentes a la composición química, no son en principio tan significativas como para provocar estos resultados tan diferentes en propiedades de impacto.

Esta disparidad de comportamiento entre ambas coladas, no se debe a la orientación de mecanizado de las probetas CVN ensayadas, ya que este mismo comportamiento se reprodujo tanto en la orientación TL como en la LT de las probetas subdimensionadas KLST. El resultado de dicho ensayo se puede observar en las figuras 9 y 10.

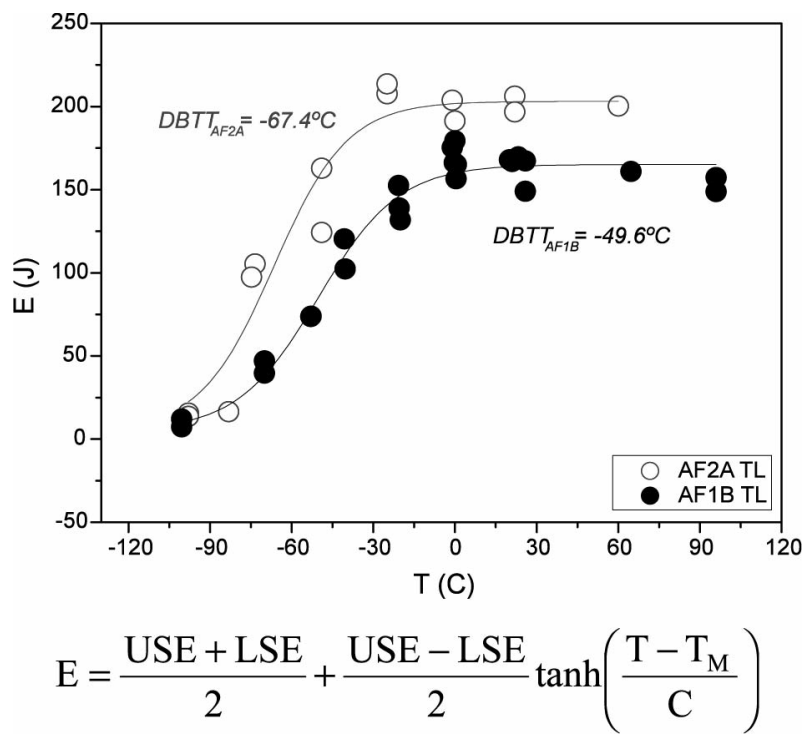

Figura 8. Curva de transición dúctil/frágil de las coladas AF1B y AF2A con probetas estándar tipo CVN.

Figure 8. Ductile/brittle transition curve for $A F 1 B$ and AF2A with CVN standard specimen.

El resultado de los ensayos con probetas no estandarizadas KLST denota no solo que la diferencia entre ambas coladas existe en ambas orientaciones, sino que además esta diferencia es mayor en la orientación LT.

Una vez analizadas las propiedades a impacto en ambas coladas, se analizaron las del candidato europeo EUROFER97-2 con probetas estandarizadas tipo CVN. El resultado de dicho análisis junto a su

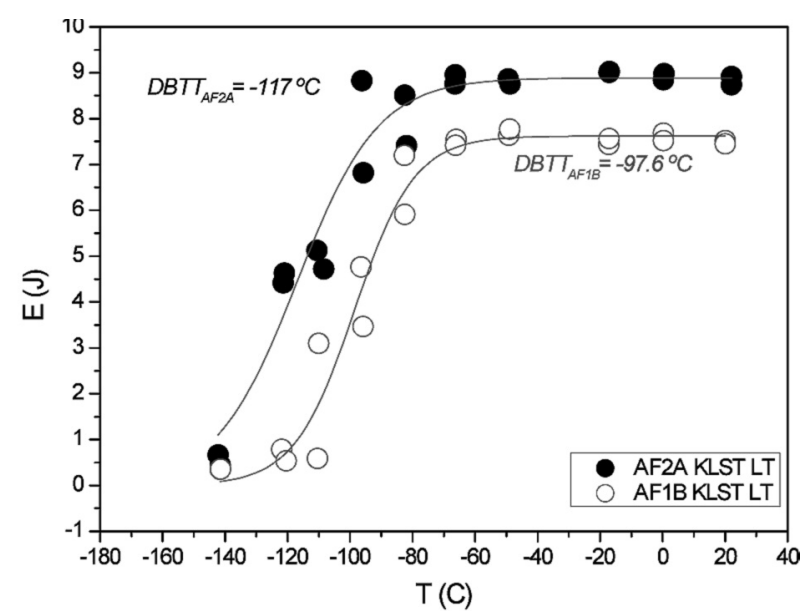

Figura 9. Curva de transición dúctil/frágil del AF1B y AF2A con probetas KLST-LT.

Figure 9. Ductile/brittle transition curve for $A F 1 B$ and AF2A with specimen KLST-LT. 
CARACTERIZACIÓN MECÁNICA DE UN ACERO FERRITICO/MARTENSÍTICO DE ACTIVACIÓN REDUCIDA DE PRODUCCIÓN ESPAÑOLA MECHANICAL CHARACTERIZATION OF A REDUCED ACTIVATION 9CR FERRITIC/MARTENSITIC STEEL OF SPANISH PRODUCTION

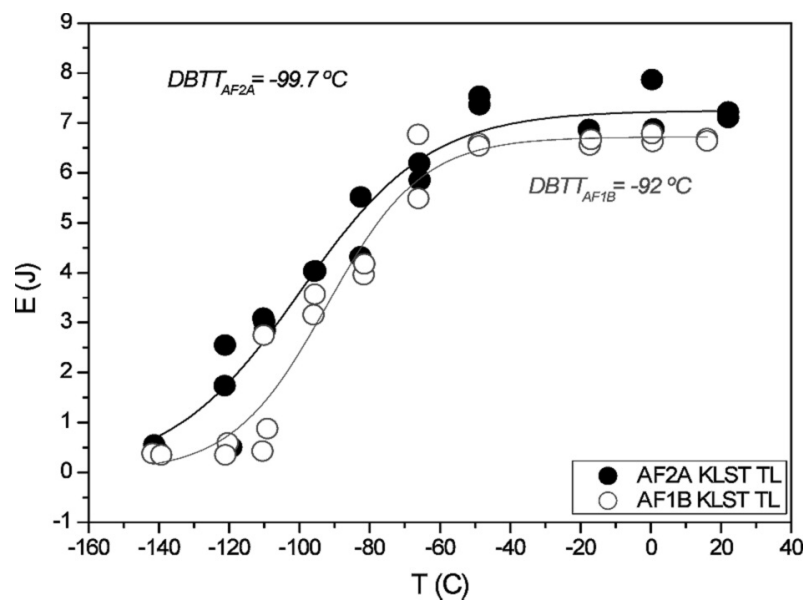

Figura 10. Curva de transición dúctil/frágil del AF1B y AF2A con probetas KLST-TL.

Figure 10. Ductile/brittle transition curve for AF1B and AF2A with specimen KLST-TL.

comparación con los resultados obtenidos para las coladas del RAFM español se pueden observar en la figura 11.

En dicha figura podemos ver cómo no solo el EUROFER97-2 posee una USE mucho mayor (un 29 \% con respecto a la colada AF2A), sino que la colada AF1B no alcanza la energía mínima de $200 \mathrm{~J}$ requerida por la especificación del EUORFER97 a $24^{\circ} \mathrm{C}$, y que la colada AF2A lo alcanza pero con dificultad, ya que hay valores a dicha temperatura que no superan dicho límite. Por el contrario, ambas coladas poseen una DBTT mucho menor que la registrada por el EUROFER97-2, en especial la colada AF2A, con la que existe una diferencia de más de $25^{\circ} \mathrm{C}$. Esto supone una ventaja considerable frente a la fragilización por radiación ${ }^{[19}$ y 20$]$.

Debido a la gran diferencia existente entre ambas coladas del RAFM español, y al no encontrar una justificación clara compartiendo el mismo proceso de producción, se realizó un análisis detallado de las superficies de fractura mediante la técnica de microscopía electrónica de barrido (SEM). En las figuras 12 y 13 podemos ver las superficies de fractura de dos probetas tipo CVN de las coladas AF1B y AF2A ensayadas en la región dúctil.

Este análisis fractográfico reveló que las probetas correspondientes a la colada AF1B presentaban una serie de inclusiones de morfología esférica y tamaño diverso. La mayoría de estas inclusiones se encontraban en el interior de los típicos huecos producidos en una fractura dúctil. A través del programa de análisis de imagen J MicroVision 1.2.7 se obtuvo que la mayoría de las inclusiones observadas presentaban

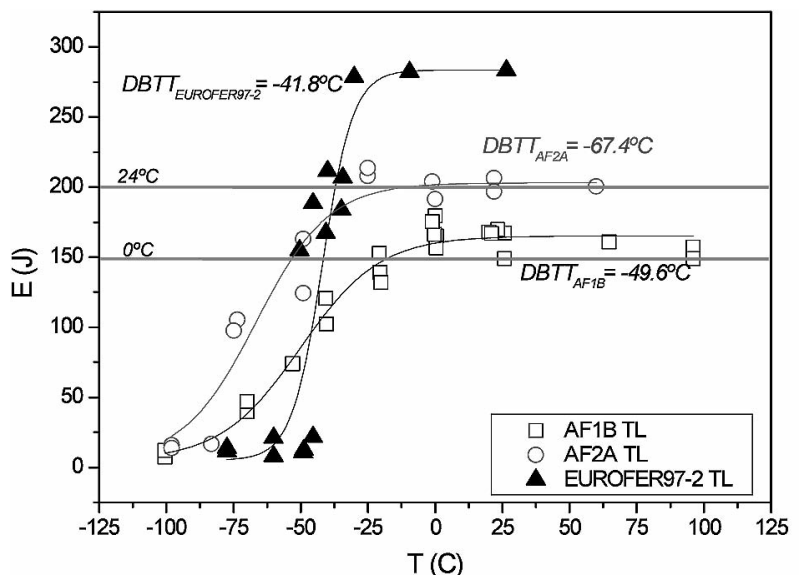

Figura 11. Curva de transición dúctil/frágil del EUROFER97-2, AF1B y AF2A con probetas estándar CVN. Límites de especificación a 0 y $24^{\circ} \mathrm{C}$.

Figure 11. Ductile/brittle transition curve for EUROFER97-2, AF1B and AF2A with CVN standard specimen. Specification's limits at 0 and $24^{\circ} \mathrm{C}$.

un tamaño comprendido entre 1 y $6 \mu \mathrm{m}$, aunque también se encontraron inclusiones con tamaños en torno a las $10-15 \mu \mathrm{m}$.

Al analizar las superficies fractográficas correspondientes a las probetas de la colada AF2A (Fig. 13), se vio que para esta colada las superficies de fractura se encontraban libres de estas inclusiones, al menos de inclusiones similares en tamaño y cantidad. Esta ausencia en las probetas de la colada AF2A, lleva a determinar que la existencia de estas inclusiones, no solo es algo exclusivo de la colada $\mathrm{AF} 1 \mathrm{~B}$, sino que posiblemente sean las responsables de la diferencia existente entre las propiedades a impacto de ambas coladas.

El tamaño considerable de las inclusiones y el que aparecieran en el interior de las fracturas dúctiles de las probetas CVN-TL, hace sospechar que sean puntos preferenciales para la nucleación de microcavidades durante ensayos a tracción. En la figura 14, se puede ver la superficie de fracturas de las probetas ensayadas a tracción de las coladas AF1B y AF2A a temperatura ambiente, y cómo efectivamente las correspondientes a la AF1B presentan unos huecos o "dimples" de gran tamaño, en cuyo interior se pueden observar aun algunas de las inclusiones que han provocado dichos "dimples"[21].

La fractografía de las probetas de tracción de la colada AF2A presenta la composición típica de una fractura dúctil en un material homogéneo, todo lo contrario de lo que ocurre en el caso de la AF1B, la cual presenta grandes "dimples", en los que podemos observar las inclusiones esféricas detectadas anteriormente y responsables de los mismos. La existencia de 

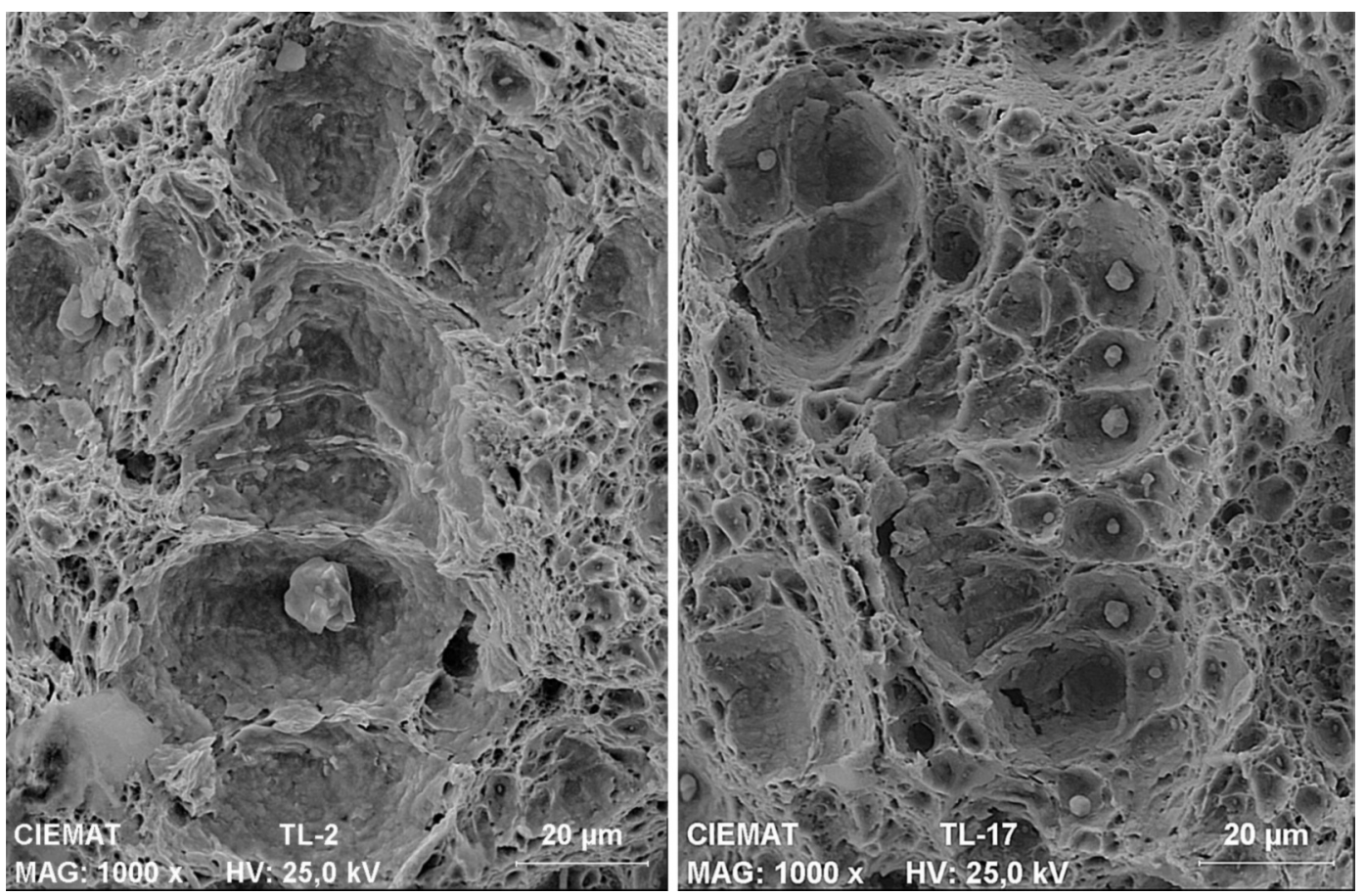

Figura 12. Superficie de fractura de probetas CVN-TL de AF1B de la región dúctil.

Figure 12. Fracture surface of AF1B CVN-TL specimens in the ductile region.

dichas inclusiones y la morfología de la fractura dan una explicación al porqué la colada AF1B poseía valores de rotura dúctil menores que los obtenidos para la colada AF2A, ya que dichas inclusiones actúan como puntos de concentración de tensiones, favoreciendo la aparición de fracturas dúctiles a cargas menores de las esperadas.

Por todo ello, y a fin de optimizar tanto el comportamiento mecánico del RAFM español, como su proceso de producción, se procedió a determinar la
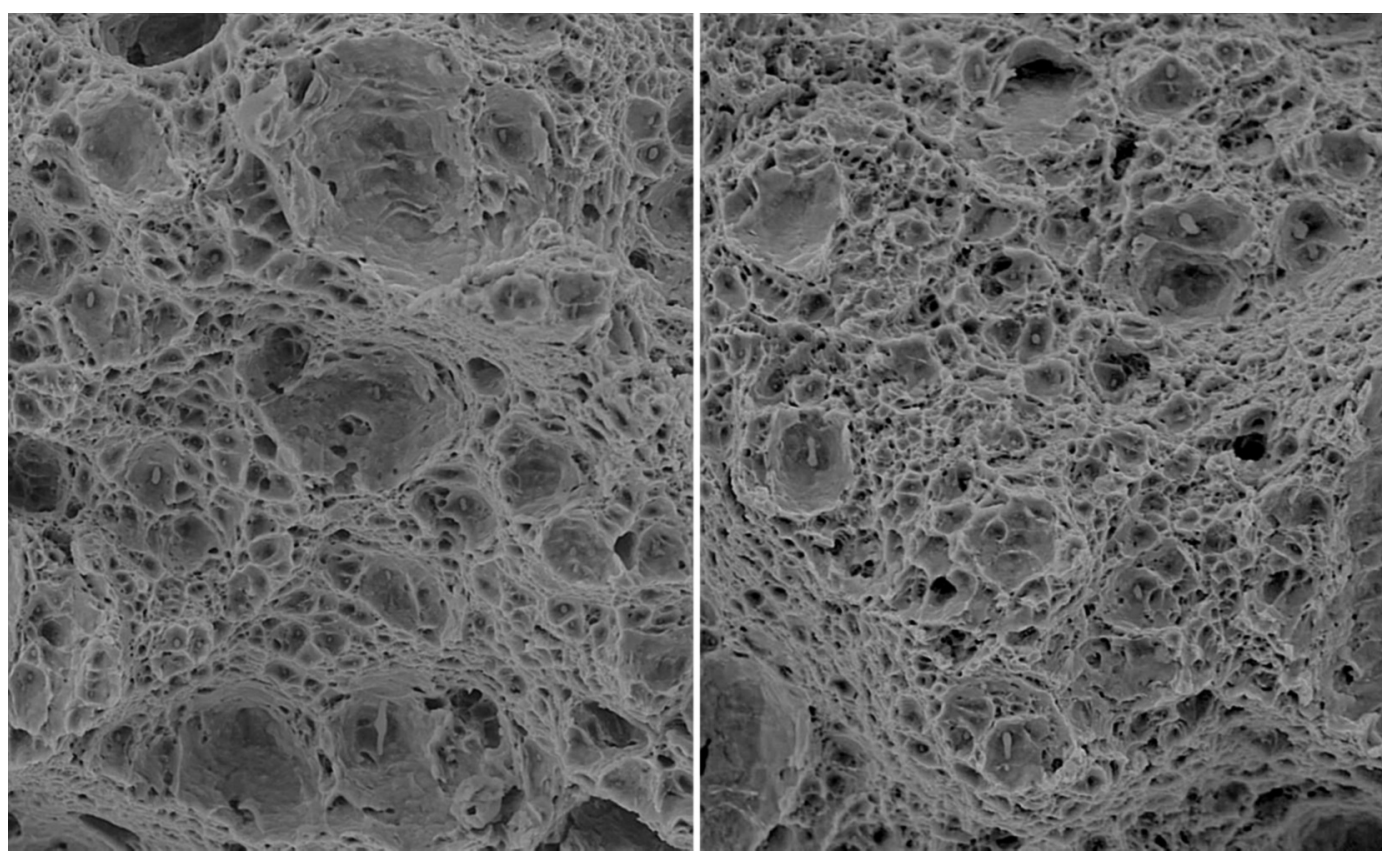

Figura 13. Superficie de fractura de probetas CVN-TL de AF2A de la región dúctil.

Figure 13. Fracture surface of AF2A CVN-TL specimens in the ductile region. 

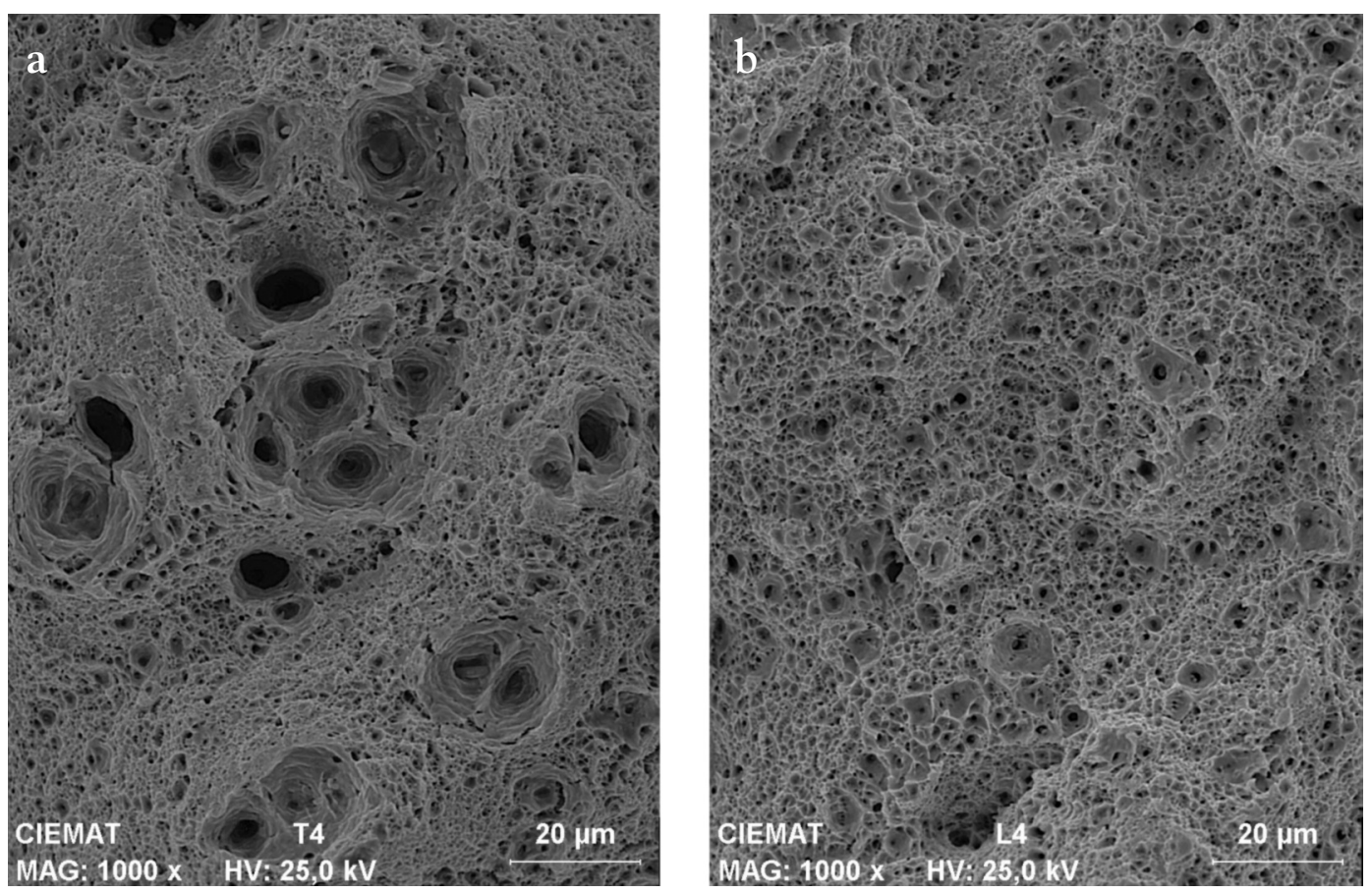

Figura14. Superficie de fractura de las probetas de tracción de M5 para las coladas: a) AF1B y b) AF2A.

Figure 14. Fracture surface of $M 5$ tensile specimens for casting: a) AF1B and b) AF2A.

naturaleza de dichas inclusiones y de este modo, el porqué de su formación.

Dicho análisis se centró en el uso de la técnica de microscopía electrónica de barrido con capacidad para emisión de campo (Scanning Electron Microscope with Field Emission Source, SEM-FEG), disponible en el Departamento de Materiales del CIEMAT.

El análisis de las muestras metalográfícas de AF1B por SEM-FEG reveló una gran cantidad de inclusiones de diverso tamaño, al igual que ocurría en las superficies fractográficas. En la figura 15 se muestra una imagen de retrodispersados, en la cual se observa cómo las inclusiones están formadas por dos fases claramente diferenciadas, una oscura con una morfología esférica y otra más brillante que se encuentra adherida a ella.

Para determinar la composición de cada una de las fases se realizaron análisis de EDX con el mismo microscopio, los cuales determinaron que las zonas oscuras de las inclusiones correspondían a óxidos de aluminio, mientras que las zonas más brillantes se trataban de óxidos de tántalo. En las figuras 16 y 17, se incluyen los espectros resultantes.

Un recorrido por la bibliografía de los RAFM revela que el profesor P. Mueller ya detectó tanto en el F82Hmod como en la primera versión del EUROFER (EUROFER97-1), la existencia de cier- tas particulas bifásicas, aunque presentaban claras diferencias entre ambos aceros. Las inclusiones bifásicas encontradas en el EUROFER97-1, poseían un tamaño máximo de partícula entorno a las $6 \mu \mathrm{m}$, por el contrario en el F82Hmod se detectaron partículas de hasta $25 \mu \mathrm{m}^{\text {[22] }}$ (Fig. 18).

El análisis de EDX de las inclusiones bifásicas encontradas en el EUROFER97-1, reveló que estas inclusiones estaban compuestas a partir de óxidos

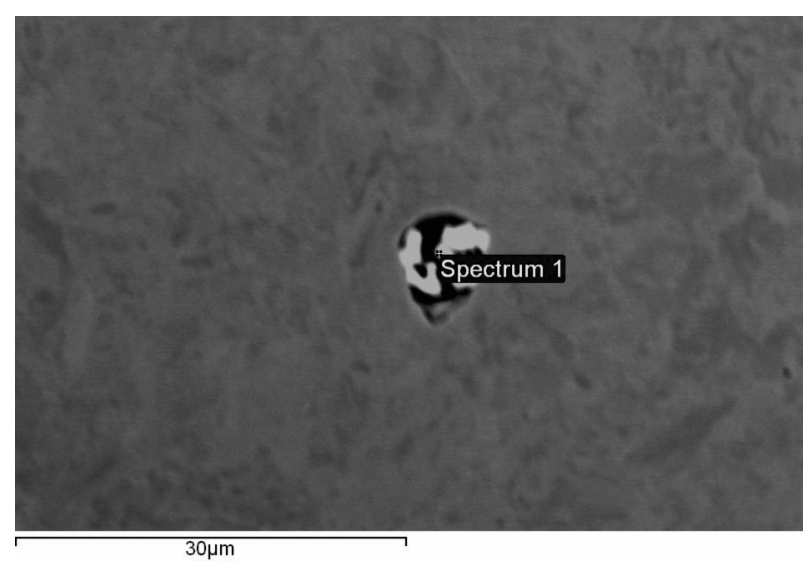

Figura 15. EBSD de una inclusión bifásica del AF1B.

Figure 15. EBSD of biphasic inclusion in $A F 1 B$. 


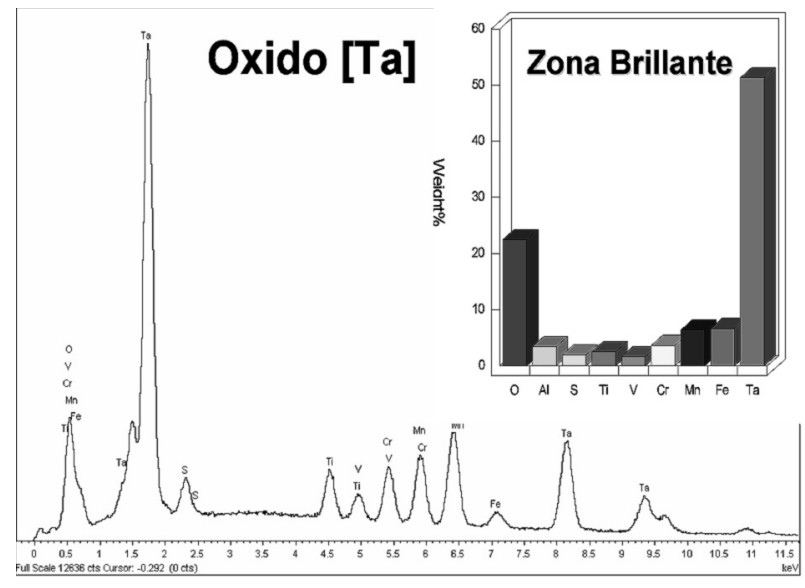

Figura 16. Espectro de EDX de la zona brillante corresponde a un oxido de tántalo.

Figure 16. EDX composition in the light region, shows the presence of tantalum oxide.

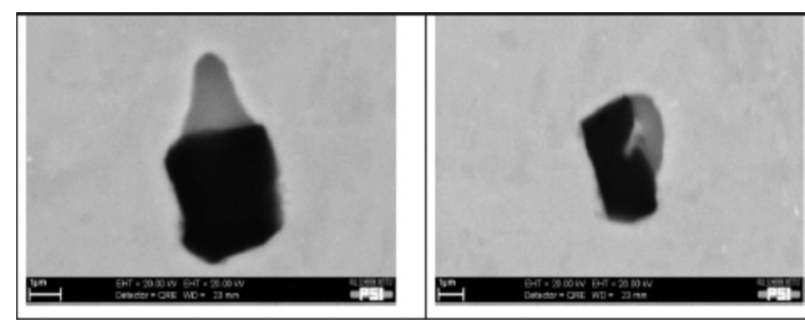

Figura 18. Inclusiones bifásicas detectadas en el EUROFER97-1 [20].

Figure 18. Biphasic inclusions detected in the EUROFER97-1 [20].

de aluminio, las cuales conformaban la mayor parte de la inclusión (zona oscura), con sulfuros de manganeso, que suponían la parte minoritaria (zona brillante). Por el contrario el análisis realizado a las inclusiones bifásicas detectadas en el F82Hmod, reflejó que la zona oscura estaba compuesta, al igual que el EUROFER97-1, de óxidos de aluminio, mientras que la zona brillante, contenía óxidos de tántalo, y no sulfuros de manganeso como en el EUROFER97-1.

Mueller realizó un sencillo cálculo basándose en la cantidad de aluminio y oxígeno que es necesario para formar $1 \mathrm{~mol} \mathrm{de} \mathrm{Al}_{2} \mathrm{O}_{3}$, según su estequiometría. Posteriormente trasladó este cálculo a la composición química obtenida en las coladas de EUROFER97-1 y F82Hmod que había estudiado, y donde aparecían las dos inclusiones bifásicas. El resultado de dicho cálculo se puede ver en la tabla VI.

En la tabla podemos ver cómo el EUROFER97-1 posee una relación $\mathrm{Al} / \mathrm{O}$ que permite al aluminio

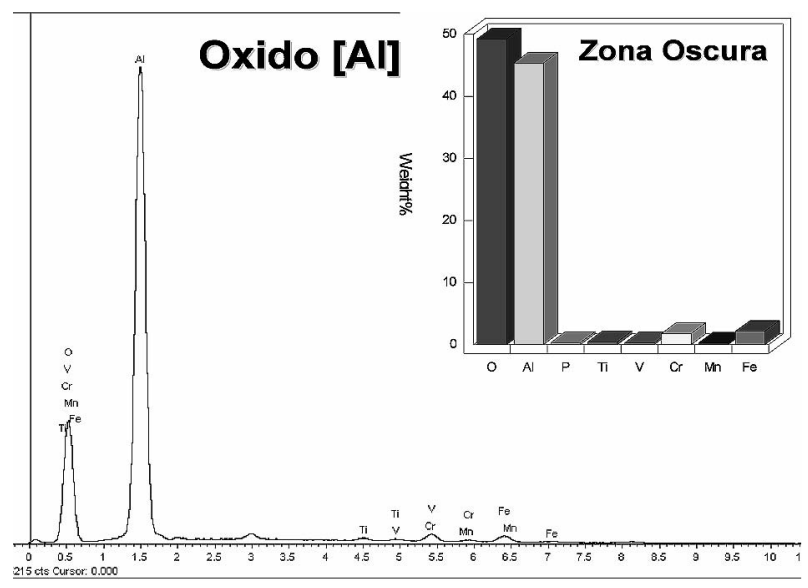

Figura 17. Espectro de EDX de la zona oscura corresponde a un oxido de aluminio.

Figure 17. EDX composition in the dark region, shows the presence of aluminium oxide.

Tabla VI. Comparación del aluminio y oxígeno necesario para formar óxidos para cada RAFM ${ }^{[21]}$

Table VI. Comparison of aluminium and oxigen needed to form oxides for each RAFM[21]

\begin{tabular}{lccc}
\hline & $\mathbf{A l}(\mathbf{g})$ & $\mathbf{O}(\mathbf{g})$ & $\mathbf{A l} / \mathbf{O}$ \\
\hline $\mathrm{Al}_{2} \mathrm{O}_{3}$ & 54 & 48 & 1,125 \\
EUROFER97-1 & 8 & 0,7 & 11,43 \\
F82Hmod & 1 & 7,4 & 0,135 \\
\hline
\end{tabular}

fijar la mayoría del oxígeno evitando de este modo que el tántalo forme óxidos. Por el contrario, el F82Hmod presenta la situación inversa, en la cual la cantidad de aluminio existente en el acero no es capaz de fijar la mayoría del oxígeno, lo que favorece la formación de los óxidos de tántalo y por lo tanto la aparición de las ya mencionadas inclusiones bifásicas de gran tamaño.

Al realizar este sencillo cálculo para las concentraciones de aluminio y oxígeno detectadas en nuestras dos coladas del RAFM español (Tabla II), obtenemos el resultado reflejado en la tabla VII.

\section{DISCUSIÓN}

El resultado de la caracterización mecánica de las dos coladas del RAFM español revela que dicho material posee unas propiedades a tracción muy parecidas a las del EUROFER97-2, cumpliendo de esta forma las exigencias mecánicas referidas a este ensayo en la especi- 
CARACTERIZACIÓN MECÁNICA DE UN ACERO FERRITICO/MARTENSÍTICO DE ACTIVACIÓN REDUCIDA DE PRODUCCIÓN ESPAÑOLA MECHANICAL CHARACTERIZATION OF A REDUCED ACTIVATION 9CR FERRITIC/MARTENSITIC STEEL OF SPANISH PRODUCTION

Tabla VII. Comparación del aluminio y oxígeno necesario para formar óxidos para cada colada del RAFM español

\section{Table VII. Comparison of aluminium and oxygen needed to form oxides for each casting of spanish RAFM}

\begin{tabular}{lccc}
\hline & Al (g) & O (g) & \multicolumn{1}{c}{ Al/O } \\
\hline $\mathrm{Al}_{2} \mathrm{O}_{3}$ & 54 & 48 & 1,125 \\
$\mathrm{AF} 2 \mathrm{~A}$ & 50 & 20 & 2,5 \\
$\mathrm{AF} 1 \mathrm{~B}$ & 10 & $<10$ & $<1$ \\
\hline
\end{tabular}

ficación del mismo. El mayor porcentaje en peso de wolframio en la colada AF1B, parece que es el responsable de una mayor UTS debido a un endurecimiento por disolución sólida, aunque normalmente los porcentajes que se barajan en este tipo de endurecimientos son algo mayores ${ }^{[24]}$.

Por el contrario, las propiedades a impacto de ambas coladas experimentan un comportamiento completamente diferente al del EUROFER97-2. La colada AF2A alcanza los $200 \mathrm{~J}$ que exige la especificación, aunque es importante reseñar que no lo hace de forma clara, ya que se registran valores a temperatura ambiente por debajo de ese límite. La colada AF1B presenta un decaimiento de las propiedades a impacto con respecto a la colada AF2A, lo que provoca que no cumpla especificaciones.

La razón del comportamiento a impacto de la colada AF1B se puede asociar a la existencia de inclusiones bifásicas de óxidos de aluminio y tántalo, observadas en la fractografía, las cuales serían las responsables de que dicha colada experimentara una energía de impacto a temperatura ambiente $18 \%$ menor que la registrada para la colada AF2A.

La formación de estas inclusiones compuestas principalmente por óxidos de aluminio se basa en la afinidad de este elemento por el oxígeno, la cual genera óxidos de una gran estabilidad, y explicaría su presencia en ambos materiales ${ }^{[21]}$.

Por otro lado la energía libre de Gibbs que posee el óxido de aluminio es $50 \mathrm{kCal} / \mathrm{mol}$ menor que la que posee el óxido de tántalo, esta diferencia provocaría que el oxígeno existente en el acero tendiera a asociarse preferentemente con el aluminio antes que con el tántalo. Esto podría dar lugar no solo la formación de los óxidos de aluminio ya mencionados, sino que el tántalo existente tendería a asociarse con el carbono para formar pequeños carburos, los cuales son los responsables de actuar como puntos de anclaje de los granos de austenita primaria, y por lo tanto, de estabilizar el tamaño de grano de los mismos en los procesos térmicos posterio- res $^{[23]}$. El problema surgiría cuando la cantidad de aluminio existente en el acero no fuera capaz de fijar la mayoría del oxígeno, ya que de este modo el tántalo tendería a formar óxidos en vez de carburos. Esto provocaría no solo un descenso en la cantidad de TaC, y por consiguiente en la estabilidad del grano austenítico primario en tratamientos térmicos posteriores, sino que los óxidos de tántalo resultantes se formarían junto a los de aluminio, generando un aumento considerable del tamaño de las inclusiones y por lo tanto un descenso de la resistencia a fractura del material ${ }^{[21]}$.

Como podemos observar en la tabla VII, en nuestro caso la colada AF2A posee una relación en peso de $\mathrm{Al} / \mathrm{O}$ por encima del límite fijado por la formación de $1 \mathrm{~mol} \mathrm{Al}_{2} \mathrm{O}_{3}$. Por el contrario la relación obtenida para la colada AF1B, no alcanza dicho límite. Al igual que ocurría para el EUROFER97-1, la cantidad de aluminio presente en la colada AF2A es suficiente para fijar la cantidad necesaria de oxígeno y evitar la formación de óxidos de tántalo, mientras que ocurre todo lo contrario en la colada AF1B, ya que como ocurría en el $\mathrm{F} 82 \mathrm{Hmod}$, la cantidad de oxígeno existente está por encima de la capacidad de fijación mediante $\mathrm{Al}_{2} \mathrm{O}_{3}$ para dicha colada, provocando que se formen los óxidos de tántalo y por tanto produzca la pérdida de resistencia a fractura experimentada en dicha colada.

\section{CONCLUSIONES}

- El estudio preliminar de la microestructura de ambas coladas experimentales revela la típica estructura de un acero $\mathrm{F} / \mathrm{M}$, con poco o ningún indicio de anisotropía debido al proceso de laminado. El tamaño de grano austenítico primario tanto para la colada AF1B como para la AF2A es más fino que el observado en el EUROFER97-2. Este estudio también revela la existencia de inclusiones de diverso tamaño y morfología en la colada AF1B, las cuales no se detectaron ni en la colada AF2A, ni en el EUROFER97-2.

- El comportamiento mecánico a tracción entre el EUROFER97-2 y las coladas del RAFM experimental son prácticamente idénticas, y todas ellas satisfacen las especificaciones dictadas por EFDA para aceros ferríticos/martensíticos de activación reducida. Por el contrario el comportamiento a impacto referido a la energía absorbida por el material a temperatura ambiente no reproduce dicha similitud, ya que ambas coladas, y en especial la AF1B, poseen una menor resistencia que la registrada para el EUROFER97-2. La causa de que la colada AF1B presente valores tan bajos con respecto a la colada AF2A puede 
deberse a la existencia de óxidos bifásicos de aluminio y tántalo. Esta desventaja de la energía de absorción entre las coladas experimentales y el EUROFER97-2 se ve suplida en cierto modo por una DBTT mucho menor, que desde el punto de vista de la fragilización por irradiación es de enorme importancia.

- Por consiguiente, para obtener unas buenas propiedades mecánicas del material, y más concretamente de impacto, se hace necesario vigilar la cantidad de oxígeno existente en el proceso de fabricación, ya que controlando la cantidad de oxígeno, se puede prevenir la formación de inclusiones de gran tamaño compuestas por óxidos de aluminio y óxidos de tántalo. Este control se basaría en mantener las concentraciones de oxígeno en niveles que permitieran al aluminio fijar la mayor parte del mismo, evitando de ese modo la formación de óxidos de tántalo. Asimismo este control permitiría que no se produjera una pérdida sustancial en la cantidad de carburos de tántalo, y por lo tanto en la capacidad de estabilización del tamaño de grano austenítico.

\section{REFERENCIAS}

[1] Memoria técnica del programa TECNO-FUS. CONSOLIDER-INGENIO 2010 Convocatoria MICINN, 2008, Madrid.

[2] E. Boom, R. W. Conn, J. W. Davis, R. E. Gold, R. Little, K. R. Schultz y D. L. Smith. J. Nucl. Mater. 122 (1984) 17-26.

[3] P. Schiller, J. Nucl. Mater. 206 (1993) 113-120.

[4] R. L. Klueh y E. Bloom, Fusion Eng. Des. 2 (1985) 383-389.

[5] R.L Klueh y D.R. Harries, High-Chromium Ferritic and Martensitic Steels for NuclearAplications. Ed. ASTM, 2001, pp. 2-23.

[6] S. J. Zinkle, International Workshop on Advanced Computational Science: Application to Fusion and Generation-IV Fission Reactors (2004).

[7] Report of IEA Workshop on Reduced Activation Ferritic/Martensitic Steels, Tokyo, Japan, 2-3 November 2000 (JAERI-Conf 2001-2007).

[8] B Van der Schaaf, F Tavassoli, C Fazio, E Rigal, E Diegele, R Lindau y G LeMarois, Fusion Eng. Des. 69 (2003) 197-203.

[9] R. Lindau, Nuclear Fusion Programme Annual Report of the Association Forschungszentrum
Karlsruhe/EURATOM. FZKA 7383, Karlsruhe (2008).

[10] H. Tanigawa, K. Shiba, A. Möslang, R.E. Stoller, R. Lindau, M.A. Sokolov, G.R. Odette, R.J. Kurtz y S. Jitsukawa, J. Nucl. Mater. 417 (2011) 9-15.

[11] N. Baluc, D.S. Gelles, S. Jitsukawa, A. Kimura, R.L. Klueh y G.R. Odette, J. Nucl. Mater. 367 (2007) 33-41.

[12] R Baldev, K. Bhanu y A.K. Bhaduri. Fusion Eng. Des. 85 (2010) 1.460-1.468.

[13] A. Morán, R. Coto, J. Belzunce y J.M. Artímez. Experimental development at a pilot plant scale of a reduced activation ferritic/martensitic RAFM steel, Asturfer ${ }^{\circledR}$. Advances in Science and Technology, 73, Switzerland, 2010, pp. 36-40.

[14] ASTM E 399-90. Standard Test Method for PlaneStrain Fracture Toughness of Metallic Materials, http://www.astm.org/DATABASE.CART/HIS. TORICAL/E399-0R97.htm.

[15] E8M-04, Standard Test Methods for Tension Testing of Metallic Materials [Metric], http://www.astm.org/DATABASE.CART/HISTORICAL/E8-04.htm.

[16] E23-02a, Standard Test Methods for Notched Bar Impact Testing of Metallic Materials. http://www.astm.org/DATABASE.CART/HISTORICAL/E23-02A.htm

[17] E112-96e3. Standard Test Methods for Determining Average Grain Size. http://www.astm.org/ DATABASE.CART/HIS. TORICAL/E112-96E3.htm.

[18] W. Dietz, A. Alamo y R. Lindau. Procurement Specification Reduced Activation Ferritic Martensitic Steel Type 9CrWTaV. European Blanket Project Structural Materials.

[19] M. Serrano, Tesis Doctoral, Escuela Técnica Superior de Ingenieros Industriales, Universidad Politécnica de Madrid, 2007.

[20] R. Viswanathan, Damage Mechanisms And Life Assessment Of High-Temperature Components. Ed. ASTM International, 1989.

[21] R. L. Stedfeld, Metals Handbook Fractography, ASTM International 12, 1985.

[22] P. F. Mueller. Tesis Doctoral, Faculté Sciences de Base, École Polytechnique Fédérale de Lausanne, Suisse, 2009.

[23] L. Xing-gang, Y. Qing-zhi, M. Rong, W. Haoqiang y G. Chang-Chun. J. Iron Steel Res. Int. 17 (2010) 57-62.

[24] R. H. Perry, Chemical Engineers' Handbook, Sixth Edition. Ed. McGraw-Hill, 1987. 